\title{
APERTURA COMERCIAL Y DISTRIBUCIÓN DE LA RENTA EN PAÍSES EN DESARROLLO Un análisis de la experiencia de Irlanda
}

\author{
JUAN RAFAEL MORILLAS MARTÍNEZ \\ Universidad de Oxford (Reino Unido) y CEACS (Instituto Juan March)
}

PALABRAS CLAVE ADICIONALES

Sociología del desarrollo, Liberalización comercial, Distribución de la renta, Irlanda.

\section{ADDITIONAL KEYWORDS}

Sociology of development, Trade openness, Income Distribution, Ireland.

RESUMEN. En este trabajo se analizan los efectos distributivos de las estrategias de desarrollo basadas en la promoción de las exportaciones y la atracción de inversiones extranjeras. En concreto, se analizan las consecuencias de la estrategia de desarrollo seguida en Irlanda. Una larga tradición en economía y sociología del desarrollo ha mantenido que la apertura comercial tiene consecuencias igualitarias sobre la distribución de la renta en los países en vías de desarrollo. En este trabajo presento los fundamentos teóricos de esta afirmación y los discuto a la luz de las críticas realizadas por nuevas teorías del desarrollo. El análisis de la experiencia de Irlanda constituye un caso especialmente adecuado para probar empíricamente las proposiciones derivadas de ambas teorías.

ABSTRACT. In this paper I analyze the distributive effects of development strategies based on export and direct foreign investment. Specifically, I analyze a case study: the distributive effects of the development strategy followed by Ireland since the 1960s. A long tradition in the Economics and Sociology of Development has maintained that trade openness has equalitarian distributive effects in developing countries. In this paper I present the theoretical foundation of such a statement and discuss its plausibility in the light of the criticisms raised by new theories of development. The Irish experience provides an extremely appropriate case study to test the propositions derived from both sorts of theories.

E-mail: juanrafael.morillas@nuffield.oxford.ac.uk

Revista Internacional de Sociología (RIS)

Tercera Época, nº 31, Enero-Abril, 2002, pp. 83-111. 
R I S

REVISTA INTERNACIONAL DE SOCIOLOGí

$N^{0}$ 31, Enero-Abril, 2002

JUAN RAFAEL MORILLAS MARTÍNEL

\section{INTRODUCCIÓN}

"The rising tide that will rise all our boats"

La frase que inicia estas páginas era común en las discusiones de políticos y científicos sociales irlandeses sobre la nueva estrategia de industrialización adoptada en los últimos años de la década de los cincuenta. La estrategia de industrialización seguida por Irlanda desde la consecución de la independencia política consistió en la promoción de la industrialización mediante la sustitución de importaciones (en adelante ISI). La evolución de sus resultados económicos determinó el abandono de la misma a finales de los años cincuenta y la adopción de una nueva estrategia orientada a la promoción de las exportaciones y a la atracción de inversiones extranjeras directas (en adelante ELI). La frase inicial expresa la confianza de economistas y políticos en que esta estrategia generaría unos efectos socioeconómicos favorables que no se limitarían a los sectores directamente fomentados por ella, sino que se extenderían a toda la economía y la sociedad.

El crecimiento económico conseguido desde entonces en Irlanda ha sido muy considerable. Aunque la promoción de las exportaciones y la atracción de "inversiones extranjeras directas" (en adelante FDIs) han sido constantes desde finales de los años cincuenta, esta estrategia ha tenido dos orientaciones. En los años sesenta las FDIs se orientaron a sectores intensivos en trabajo, atraídas, además de por los incentivos fiscales concedidos por el Estado irlandés, por los bajos costes laborales de este país. Desde la primera mitad de los setenta, la agencia estatal encargada de la política industrial orientó su estrategia a la atracción de FDIs en sectores de alta tecnología. Los resultados económicos han sido especialmente favorables desde entonces, obteniendo tasas de crecimiento equiparables a las de los países en vías de industrialización (en adelante NICs) del Este asiático. Pero los resultados económicos también han resultado llamativos por la evolución no tan favorable de otras variables. La tasa de desempleo y el porcentaje de desempleados de larga duración han sido muy altos y mayores que la media de la Unión Europea. La estructura económica se ha caracterizado por un marcado carácter dual. Se ha creado un sector moderno y dinámico, mayoritariamente de propiedad extranjera - resultado de las FDIs, que han incorporado tecnologías muy avanzadas-, y ha coexistido con un sector tradicional doméstico caracterizado por bajos niveles de productividad, escaso desarrollo técnico, baja competitividad y sin vinculación al sector moderno.

En este trabajo analizo la experiencia de industrialización seguida por Irlanda desde finales de los cincuenta, con especial énfasis en la fase posterior a 1973. El análisis está orientado por los argumentos de las "nuevas teorías" del comercio internacional y por un modelo analítico que desarrollo a partir de tales argumentos. La elección de este caso de estudio está determinada porque su 
proceso de industrialización se ha ajustado a las características de la estrategia ELI. Como ha señalado O'Malley «aunque un número considerable de países en desarrollo ha adoptado ciertos elementos de esta estrategia, tales como los objetivo de promoción de las exportaciones y los incentivos para atraer empresas extranjeras [...] pocos han llegado tan lejos como Irlanda» (1992: 49).

En una primera sección del trabajo discuto las causas y consecuencias de las estrategias de industrialización seguidas en los países en desarrollo. En concreto, parto de la erosión de la confianza en la estrategia ISI y de la creciente afirmación de la idoneidad de la adopción de estrategias ELI para promover el desarrollo industrial. Presento los fundamentos teóricos de la afirmación de los efectos distributivos de signo igualitario de esta estrategia y los discuto a la luz de las advertencias realizadas por las "nuevas teorías", que subrayan la complejidad de los fenómenos implicados.

En segundo lugar, presento un modelo analítico cuyo propósito es describir e ilustrar las implicaciones distributivas de algunas variables características de la estrategia ELI. Incorporo en él las consideraciones de las "nuevas teorías" y otras adicionales que permitan facilitar la ilustración de las consecuencias distributivas de esta estrategia.

En tercer lugar, estudio la experiencia de Irlanda. Describo las fases que ha seguido su proceso de industrialización desde la consecución de la independencia política. Me centro en los resultados socioeconómicos de la estrategia ELI, especialmente en la fase posterior a 1973. Centro el análisis en la evolución de las variables ligadas a las consideraciones teóricas de las "nuevas teorías" y las relaciones establecidas en el modelo analítico. Así, estudiaré el crecimiento de la producción industrial, la evolución del empleo, la productividad, las relaciones entre crecimiento de la producción industrial y evolución del empleo, la evolución del desempleo de larga duración y las diferencias existentes entre los sectores moderno y tradicional de la estructura económica. Tras esta descripción analizo y discuto las consecuencias distributivas de tal estrategia a la luz del modelo analítico presentado en la sección anterior.

\section{LAS ESTRATEGIAS DE INDUSTRIALIZACIÓN DE LOS PAÍSES EN DESARROLLO Y SUS CONSECUENCIAS DISTRIBUTIVAS}

La estrategia de industrialización predominante en los países en vías de desarrollo tras la Segunda Guerra Mundial fue la ISI. Esta estrategia consistió en el fomento de la industria nacional mediante la limitación de su exposición a la competencia internacional. Gráficamente, su propósito fue «la promoción de la industrialización tras un muro de protección implícita o explícita» (Findlay, 1988: 84). Para ello se utilizó una serie de mecanismos con el fin de que los productos importados no fueran competitivos en el mercado nacional. 
RIS

REVISTA INTERNACIONAL DE SOCIOLOGIA

No 31, Enero-Abril, 2002

Consistieron en la imposición de aranceles, el establecimiento de contingentes y la sobreevaluación del tipo de cambio.

La defensa de esta estrategia se fundamentó en dos tipos de argumentos. En primer lugar, el argumento de la infant-industry y de la existencia de "barreras de entrada". Las industrias de los países desarrollados y de los países en desarrollo partían de niveles de desarrollo muy diferentes. Para conseguir su equiparación era imprescindible proteger la industria de los países en desarrollo. Si no se hiciera, la competencia reproduciría de modo crónico las diferencias existentes. En segundo lugar, los efectos de la ventaja comparativa. Razonando de un modo ricardiano, se pensó que la apertura comercial conduciría a una continua especialización. Los países desarrollados se especializarían en la producción de bienes manufacturados y los países en desarrollo en la producción de productos primarios. Esa especialización produciría un aumento de la renta en ambos grupos de países. Dado que alcanzado cierto nivel de renta la elasticidad-renta de los productos importados es mayor que uno, los aumentos de renta conseguidos con la exportación de productos primarios por los países en desarrollo conduciría a un aumento proporcionalmente mayor de las importaciones de productos manufacturados (Krueger, 1988: 359). La apertura comercial, por tanto, conduciría a una especialización continua e imposibilitaría la industrialización de los países en desarrollo.

Los resultados de la estrategia ISI no fueron los esperados. Produjo un deterioro de los términos de intercambio debido a la diferente evolución de los precios de los productos exportados e importados por los países en desarrollo. En cuanto a los efectos sobre la organización industrial, la intensidad en capital de la industria aumentó considerablemente. La relación expresada por la ratio capital-output experimentó un rápido aumento (ICORs: Incremental Capital-Output Requierements). El aumento de los costes de producción de las industrias de los países en desarrollo, debido al pequeño tamaño de los mercados nacionales, incentivó el aumento de la inversión. Los aumentos de la producción no estuvieron acompañados por aumentos del empleo en el sector industrial (Krueger, 1988: 359). Las implicaciones para el mercado de trabajo no se limitaron a las dificultades de crecimiento del empleo. La segmentación del mercado de trabajo aumentó como consecuencia de los requerimientos de trabajo cualificado por las industrias intensivas en capital, del desarrollo de sindicatos con fuerte poder de mercado y del aumento de la desigualdad salarial entre insiders y outsiders (Haggard, 1990: 224-225).

Desde mediados de los años sesenta, la idoneidad de la estrategia ISI fue cuestionada. Sus crecientes dificultades y el progresivo éxito de los NICs del Este asiático fueron los motivos principales. Los argumentos a favor de la estrategia ELI han aumentado. Desde una perspectiva macroeconómica se ha enfatizado las ventajas y beneficios del comercio y de las inversiones como motor 
del crecimiento económico. Los argumentos microeconómicos han destacado los incentivos que aporta esta nueva estrategia para mejorar la competitividad, utilizar el factor de producción más abundante en las economías de los países en desarrollo - trabajo no cualificado - y superar las ineficiencias debidas a las consecuencias monopolísticas de la estrategia ISI (Krueger, 1988: 362; Haggard, 1990: 12).

Respecto a los efectos distributivos, se ha mantenido que la estrategia ELI tiene consecuencias igualitarias. Los incentivos para desarrollar actividades económicas intensivas en trabajo y la exportación de bienes con alto contenido en este factor, son la causa. El fundamento teórico de esta afirmación es aportado por los modelos neoclásicos del comercio internacional (Krugman y Obstfeld 1995: caps. 2 y 4 ).

La teoría Heckscher-Ohlin es la principal base teórica neoclásica donde se fundamenta la afirmación de los efectos distributivos de signo igualitario del comercio internacional. De acuerdo con esta teoría, la fuente del comercio internacional, la causa de su existencia, es la diferencia de dotaciones de factores entre países. Esta diferencia es consecuencia de la abundancia relativa de los factores de producción en una economía y de la tecnología de producción utilizada, que determina la intensidad relativa con que se utilizan los factores de producción. Por ello, la especialización de las economías no está determinada por las diferencias de productividad de los factores móviles, sino por las dotaciones de recursos. Así, las economías se especializan en la exportación de bienes que son intensivos en los factores en que su oferta relativa es abundante. La especialización de las exportaciones determina que el comercio internacional tenga efectos distributivos. Su influencia tiene lugar a través de dos mecanismos: el cambio de los precios relativos de los bienes, y el contenido de factores de las exportaciones de bienes. La especialización permite una reducción del precio de los bienes. Esta reducción determina que aumente la demanda del bien y, por tanto, la demanda de los factores que contiene su producción. Así es como entra en juego la influencia del efecto del contenido de factores. Este aumento de la demanda de dichos factores hace que disminuya la oferta efectiva del factor más utilizado en las exportaciones, con lo cual la renta apropiada por ese factor aumenta.

Por tanto, de acuerdo con esta teoría, el comercio internacional tiene efectos distributivos de signo igualitario para los países en desarrollo. Estos países se especializarán en la exportación de bienes que contengan el factor más abundante en su economía: trabajo no-cualificado. Ello determinará una disminución de la oferta efectiva del mismo y, como consecuencia, un aumento de su precio: las rentas apropiadas por este factor de producción aumentarán. Mientras, la situación de los trabajadores no cualificados en los países desarrollados empeorará. El comercio internacional determinará que estos países se especialicen en la producción de bienes intensivos en capital y 
RIS

REVISTA INTERNACIONAL DE SOCIOLOGIA

No 31, Enero-Abril, 2002

JUAN RAFAEL MORILLAS MARTÍNEZ

en trabajo cualificado, pues son los factores de producción los que suman la mayor parte de la dotación total de factores. Así, la demanda de trabajadores no cualificados disminuirá y, como consecuencia, la renta apropiada por este factor también descenderá ${ }^{1}$.

La afirmación de los efectos agregados y distributivos positivos del comercio internacional y de la adopción de estrategias ELI ha sido cuestionada por las "nuevas teorías" del comercio internacional. Éstas mantienen que los supuestos de que parten las teorías neoclásicas en que se fundamenta tal afirmación son demasiado restrictivos. Las teorías neoclásicas suponen que existe igual tecnología en los países implicados en el comercio y que los flujos comerciales están determinados por las diferentes dotaciones de factores. Ello implica la suposición de que las funciones de producción son homotéticas entre los diferentes países implicados en el comercio internacional: los coeficientes de los inputs y los outputs son iguales en ambos países (Lawrence, 1996: 53-58; Berry, 1992: 47).

En efecto, como ha señalado Helleiner, «los modelos (neoclásicos) en que se basa son desafiados, y la evidencia empírica sobre el impacto de tal reforma es limitada» (Helleiner, 1992: 3). Estas teorías y modelos olvidan la enorme influencia de otros fenómenos implicados en las estrategias ELI cuyas consecuencias agregadas y distributivas son de signo contrario. La orientación a la promoción de las exportaciones y la atracción de FDIs tienen consecuencias en diversos aspectos de la estructura económica que hacen más compleja la determinación de los aspectos distributivos de esta estrategia de industrialización. A saber: los cambios de productividad de los factores como consecuencia del aumento de las diferencias de intensidad en capital y en trabajo cualificado de diferentes sectores de la economía (Pack, 1992); la modificación de la organización industrial y el desarrollo de economías de escala en determinados sectores intensivos en capital (Berry, 1992); los cambios de composición de la demanda de trabajo cualificado y de trabajo no cualificado; las características de la estructura de los mercados (imperfecciones en su funcionamiento, papel de la inversión extranjera directa, etc) (Lee, 1992); el papel de las externalidades y los linkages establecidos entre los diferentes sectores de la economía (Stewart y Ghani, 1992). Todos estos fenómenos determinan que las consecuencias distributivas de la estrategia ELI no puedan ser determinadas de un modo inmediato por ecuaciones que incluyan solamente las variables dotación de recursos, precios relativos de los bienes exportados y contenido de factores de dichos bienes.

\footnotetext{
${ }^{1}$ La influencia del comercio con los países en desarrollo sobre la posición de los trabajadores menos cualificados en los países desarrollados ha generado un gran número de estudios empíricos. Para una aproximación al estado de la cuestión véase Freeman (1995) y OECD (1997b).
} 


\section{MODELO ANALÍTICO}

El propósito del modelo analítico que presento a continuación es facilitar la comprensión e ilustrar la influencia de la adopción de una estrategia ELI sobre la distribución de la renta. No pretendo estudiar todas las influencias que median en la relación entre apertura comercial, recepción de FDIs y distribución de la renta. Sería necesario estudiar la influencia de la evolución de las exportaciones, de las importaciones, de los precios de los bienes en los mercados internacionales, de la demanda de factores de producción, etc. El tipo de análisis más adecuado para el desarrollo de un modelo como éste sería un análisis de equilibrio general ${ }^{2}$, lo que supera el propósito de este trabajo. La pretensión del modelo no es determinar la evolución de las diferentes variables implicadas, sino analizar de un modo más exploratorio e ilustrativo que explicativo la tendencia general de dicha evolución. Entre las variables implicadas se considerarán las siguientes: los sectores a que se orientan las FDIs, el contenido de factores de las exportaciones, la productividad marginal de los factores en cada sector de la economía y la demanda de factores de producción.

En primer lugar, establezco unas consideraciones generales sobre la distribución personal de la renta. Partiendo de la relación entre distribución personal de la renta, utilización de factores por cada sector de la economía y productividad marginal de los factores de producción en cada sector, establezco el modo de determinar la distribución personal de la renta. Tras ello aplico las relaciones anteriores para estudiar la influencia de una orientación particular de ELI sobre la distribución de la renta y la evolución de la desigualdad. Para ello establezco la influencia de ese tipo particular de ELI sobre la estructura de la demanda de factores, sobre la plena utilización (o el subempleo) de los factores de producción y sobre la productividad marginal de los mismos en los diferentes sectores de la economía.

Antes de presentar el modelo analítico es necesario hacer dos observaciones. Primero, que las relaciones que establezco se refieren a los resultados distributivos del mercado como consecuencia de la estrategia ELI. No considero el efecto de la intervención estatal en la redistribución de la renta, por lo que, tanto en el modelo analítico que presento, como en el estudio de caso posterior (el caso

\footnotetext{
${ }^{2}$ Bourgioun y Morrisson (1989: 20-27) desarrollan un modelo de las consecuencias distributivas de la apertura comercial, en el que consideran las influencias de la evolución de las exportaciones, la importaciones, los precios en los mercados internacionales y el empleo de factores. El modelo que presento se basa en el de estos autores.
} 
RIS

de Irlanda), no hago referencia a las características de los sistemas fiscales ni a otros mecanismos de redistribución de la renta. Segundo, que tampoco tengo en cuenta la posible influencia del nivel de desarrollo económico sobre la desigualdad de la distribución de la renta ${ }^{3}$.

\section{Primer paso: distribución personal y distribución funcional de la renta}

Sea una economía caracterizada por la existencia de $n$ individuos, $m$ factores de producción y $S$ sectores de producción. Represento mediante $i$ a los individuos (siendo $i=1,2, \ldots, \mathrm{n}$ ), mediante $j$ a los factores de producción (siendo $j=1,2, \ldots$, $\mathrm{m})$ y mediante $s$ a los sectores de producción $(s=1,2, \ldots, \mathrm{S})$. Sea $D_{j}$ la dotación total de factor $j$ en la economía.

La distribución personal de la renta en esta economía estará determinada por la distribución funcional de la renta (la parte de renta apropiada por cada uno de los factores de producción) y por la estructura de la propiedad de los factores de producción.

Sea $y_{i}$ la renta del individuo $i(i=1,2, \ldots, \mathrm{n})$. Sea $a_{i j}$ la cantidad de factor $j$ que posee el individuo $i$. Sea $w_{j}$ la renta del factor $j$ según la distribución funcional de la renta. La renta del individuo $i$ estará determinada por la siguiente expresión:

$$
\text { (1) } y_{i}=\sum a_{i j} w_{j}
$$

La distribución funcional de la renta puede estar determinada de diferentes modos. Suponiendo que los mercados de factores funcionan de un modo perfectamente competitivo, la parte de renta apropiada por cada factor será una función de la productividad marginal del mismo en cada sector de la economía y de la cantidad de cada factor que emplea dicho sector. Así se expresa en la siguiente ecuación:

$$
\text { (2) } w_{j}=w_{j}\left(F_{s j} ; L_{s j}\right)
$$

\footnotetext{
${ }^{3}$ Las hipótesis de Kuznets (1955) sobre la existencia de una relación entre nivel de desarrollo económico y desigualdad en la distribución de la renta en forma de U invertida -la desigualdad es muy reducida en niveles de desarrollo económico bajos, aumenta en las primeras fases del desarrollo económico, y posteriormente disminuye una vez alcanzado un cierto nivel de desarrollo económico-, han generado un importante debate y un gran número de estudios empíricos. Para una revisión de la literatura al respecto, véase Fields (1988). Callan y Nolan (1992) tienen en cuenta esta hipótesis de Kuznets en su estudio de la distribución de la renta en Irlanda. Para ello, sitúan la experiencia de Irlanda en dos contextos: los países desarrollados y los países en desarrollo.
} 
donde $s=1,2, \ldots, \mathrm{S} ; F_{s j}$ es la productividad marginal del factor $j$ en el sector $s ; L_{s j}$ es la cantidad de factor $j$ empleado en el sector $s$. Por tanto, la renta del factor $j$ depende de su productividad marginal en cada uno de los $s$ sectores de la economía, y del nivel de empleo de dicho factor en cada uno de los sectores. Sea el nivel de empleo efectivo del factor $j$,

$$
\text { (3) } \hat{L_{j}}=\Sigma L_{s j}
$$

que se obtiene sumando la cantidad de factor $j$ empleado en cada uno de los $s$ sectores.

$$
\hat{L_{j}} \leq D_{j}=\sum_{i} a_{i j}
$$

O sea, el nivel de empleo efectivo del factor $j$ será menor o igual a la dotación total de factor $j$ de la economía, que es igual a la suma de las cantidades de factor $j$ que posee cada uno de los $i$ individuos de la economía. Introduciendo (3) en (2), se concluye que

$$
\text { (4) } \quad w_{j}=w_{j}\left(F_{s j}, \hat{L}_{j}\right) \quad j=1,2, \ldots, \mathrm{m} ; \quad s=1,2, \ldots, \mathrm{S}
$$

O sea, la renta del factor $j$ es una función de la productividad del mismo en cada uno de los $s$ sectores de la economía en que está empleado, y del nivel de empleo efectivo del mismo.

Gracias a la ecuación (1) se puede calcular la distribución personal de la renta. Para ello es necesario conocer la distribución de la propiedad de los factores y la renta de cada uno de los factores. La expresión (1) se puede plantear del siguiente modo:

$$
\text { (5) } Y=A W
$$

donde $Y$ es un vector que representa la renta de cada uno de los $i$ individuos de la economía; es un vector de orden $n \times 1$, es decir, $Y=\left(y_{1}, y_{2}, \ldots, y_{n}\right)$ donde $y_{i}$ representa la renta personal del individuo $i$.

$A$ es una matriz de orden $\mathrm{n} \times \mathrm{m}$, donde cada uno de los $a_{i j}$ elementos representa la cantidad de factor $j$ que posee el individuo $i . W=\left(w_{l}\right.$, $\left.w_{2}, \ldots, w_{m}\right)$ es un vector de orden $\mathrm{m} \times 1$, donde cada $w_{j}$ representa la renta del factor $j$ según la distribución funcional de la renta en la economía. 


\section{RIS}

Gracias a las expresiones (1) y/o (5) es posible obtener la distribución personal de la renta una vez conocidas la distribución de la propiedad de los factores y la distribución funcional de la renta ${ }^{4}$.

\section{Segundo paso: ELI y desigualdad de la distribución de la renta}

Imaginemos una economía que tiene dos sectores de producción, $S=2$ ( $s=1$, sector tradicional, y $s=2$, sector moderno). Tres son los factores utilizados en la producción de bienes $(m=3)(j=1$ capital, $j=2$ trabajo cualificado, $y j=3$ trabajo no cualificado) ( $n$ es el número de individuos de la misma).

Esta economía aumenta su stock de capital como consecuencia de la recepción de FDIs orientadas al sector moderno, en concreto a la exportación de bienes cuyo contenido de factores es alto en capital y trabajo cualificado y nulo en trabajo no cualificado. Las relaciones, linkages y externalidades positivas de las FDIs con el sector tradicional, son prácticamente nulas.

La demanda de factores según los sectores económicos es la siguiente:

- Sector tradicional: capital y trabajo no cualificado (o sea, según las expresiones del apartado anterior, $L_{12}=0$ ).

- Sector moderno: capital y trabajo cualificado (o sea, $L_{23}=0$ ). Es un sector intensivo en capital y en trabajo cualificado, orientado a la exportación de bienes que contienen los factores citados. Por tanto, la productividad marginal de este sector es muy alta, tanto en términos absolutos como en comparación con la productividad marginal del sector tradicional.

La influencia de la estrategia ELI sobre el sector tradicional es la siguiente. Es incapaz de competir con las importaciones de bienes en el mercado nacional

\footnotetext{
${ }^{4}$ El conocimiento de la estructura de la propiedad de los factores es complejo. Se podría aproximar por la estructura ocupacional de la economía. Para simplificar el examen analítico voy a suponer que la estructura ocupacional coincide con la distribución de los factores de producción en cada uno de los $s$ sectores de la economía. De este modo:

$$
\text { (6) } I=\sum p_{s} I_{s}+I\left[\hat{y_{s}} ; n_{s}\right] ; s=(1,2, \ldots, S) \text {. }
$$

Donde $I$ representa la desigualdad total, $I_{s}$ la desigualdad interna a cada grupo, y $p_{s}$ es un parámetro que representa el peso de la desigualdad interna al grupo $s$ en la desigualdad total; es una función del número de individuos que incluye el sector. $\hat{y}_{s}$ representa la renta media en el sector $s$ y $n_{s}$ representa el número de personas ocupadas en el sector $s$. La desigualdad total es la suma de la desigualdad interna en cada $y$ de la desigualdad existente entre las rentas medias en cada uno de los $s$ sectores. Presento esta ecuación para ilustrar una aproximación sectorial al estudio de la desigualdad de la distribución de la renta, aunque no la aplico al estudio de caso por desconocer los valores de las variables que incluye. Esta expresión está tomada de Borugioun y Morrisson (1989: 27).
} 
y de exportar sus productos a los mercados internacionales, debido a su bajo nivel de desarrollo técnico. La productividad marginal del sector es muy baja. Las dificultades del sector le han conducido a disminuir la demanda de trabajo no cualificado: disminuye $L_{13}$, mientras la oferta permanece constante. Por tanto, existe subempleo de $j=3$ (trabajo no cualificado). El capital empleado en este sector empeora su situación; la productividad marginal del capital en el sector tradicional disminuye con la apertura económica debido a que la pérdida de mercado nacional que experimenta no es compensada con una ganancia de mercados nacionales, y porque no está conectado con el sector moderno de la economía. La producción del sector tradicional está compuesta principalmente por la producción de bienes para los que existe un grado de protección natural significativo. El sector tradicional crece solamente en períodos en que la demanda doméstica es boyante.

La situación del sector moderno con la estrategia ELI se caracteriza por la recepción de FDIs orientadas a sectores high-tech. La productividad marginal de este sector es muy alta. Este sector es el principal responsable del crecimiento económico.

La evolución del crecimiento de la producción, del crecimiento del empleo cualificado y del empleo no cualificado seguirá la pauta representada en la figura adjunta. La causa principal del crecimiento económico es el aumento de exportaciones del sector moderno. El contenido de las exportaciones es capital y trabajo cualificado, y su alta intensidad en capital determina que los aumentos de producción coexistan con pequeños aumentos del empleo. El pequeño aumento del empleo que se produzca como consecuencia del crecimiento económico asociado al aumento de las exportaciones estará compuesto exclusivamente por empleo cualificado. La evolución del empleo no cualificado estará determinada por la evolución de la demanda de trabajo del sector tradicional, que será consecuencia de la contribución del mismo al crecimiento de la producción,

Figura 1.

Evolución a lo largo del tiempo de la producción industrial $(P)$, del empleo cualificado $(C)$ y del empleo no cualificado $(N)$

Eje X: t (tiempo)

Eje Y: P, C, N

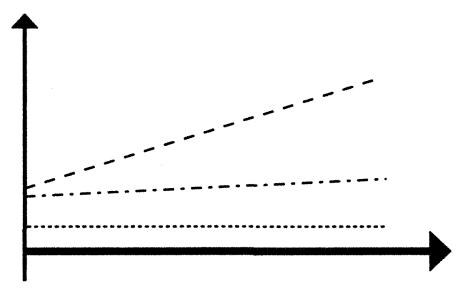


RIS

REVISTA INTERNACIONAL DE SOCIOLOCIA

№ 31, Enero-Abril, 2002

JUAN RAFAEL MORILLAS MARTINEL

pues el sector moderno no utiliza trabajo no cualificado. En períodos en que la demanda doméstica es alta aumentará algo el empleo no cualificado. La sub-utilización de trabajo no cualificado es constante, y disminuye en períodos de aumento de la demanda.

Como se observa en la figura, los crecimientos de la producción industrial son muy altos. Por ello, la pendiente de su función es muy alta. Los aumentos del empleo cualificado asociados a ese aumento de la producción industrial son mucho menores. Y el aumento del empleo no cualificado es nulo. $\mathrm{O}$ sea, $d P / d t>$ $d C / d t>d N / d t$, la pendiente de la función empleo cualificado es menor que la de producción industrial, y mayor que la del empleo no cualificado, que es igual a cero $(d N / d t=0)$, lo cual refleja que los aumentos de producción no producen aumento del empleo no cualificado.

Una vez conocidas las características de esta economía y de su estrategia ELI, es posible estudiar las tendencias de la distribución de la renta y la desigualdad mediante las ecuaciones presentadas anteriormente. En el epígrafe siguiente aplico las consideraciones establecidas en este modelo analítico al estudio de un caso y procedo a la formalización de estas relaciones.

\section{ESTUDIO DE CASO: LA EXPERIENCIA ELI EN IRLANDA}

\section{Fases de industrialización}

El sector industrial que heredó el Estado independiente irlandés fue muy modesto. La población activa empleada en el sector industrial en 1926 era el $13 \%$, y sólo el 10\% en el sector manufacturero (O`Malley, 1992: 31). Además, la producción de productos primarios elaborados representaba dos tercios de la producción total del mismo (Kennedy, 1992: 12, 13). La promoción del desarrollo industrial consistió en la adopción de una estrategia ISI. Los mecanismos utilizados fueron la subida del arancel, el establecimiento de contingentes a la importación de bienes y la concesión de ayudas y subvenciones a la industria nacional para fomentar su desarrollo. El principal instrumento utilizado fue la subida del arancel. En 1936 se elevó hasta alcanzar el 45\%, y aunque se redujo al $35 \%$ en 1938, el arancel en Irlanda fue el mayor de los países europeos en los años treinta y cuarenta (con la excepción de Alemania y España), el doble del arancel de Estados Unidos y la mitad mayor que el del Reino Unido (Haughton, 1995: 30). Las ayudas y subvenciones para fomentar la industria nacional fueron otro instrumento de gran importancia. Las principales medidas al respecto fueron el requerimiento de que la propiedad de las industrias manufactureras fuera mayoritariamente irlandesa, establecido en la Control of Manufactures Act (1932), la creación de la Industrial Credit Corporation y la promulgación de la Trade Loans Act, cuyos objetivos 
fueron facilitar créditos a iniciativas industriales nacionales (Haughton, 1995: 30, 31).

Los resultados económicos de la estrategia ISI permitieron conseguir una rápida expansión industrial durante los años treinta y cuarenta, con la sola interrupción del período de la Segunda Guerra Mundial. Así, la población empleada en el sector industrial en 1951 representó un $22 \%$ de la población activa. La producción industrial aumentó en todo el período, y dibujó la siguiente pauta de crecimiento (Haughton, 1995: 30-36). Entre 1931 y 1936 experimentó un enorme crecimiento: un 40\%. En el período 1936-38 el crecimiento fue de un modesto 4,5\%. Y en el período 1938-43 la producción industrial experimentó una importante disminución. Las causas de la misma fueron las dificultades para importar los inputs que requería la producción industrial como consecuencia de las dificultades del período de la Segunda Guerra Mundial. Además, las exportaciones disminuyeron en este período y se redujeron a la mitad (Haughton, 1995: 36). Los problemas de la producción industrial en este período demostraron las dificultades de la estrategia de autosuficiencia.

El examen de algunos datos cualitativos sobre las características del crecimiento industrial ofrece más elementos para evaluar las consecuencias de la estrategia ISI. La producción industrial se limitó a bienes de consumo y, en una medida mucho menor, a bienes intermedios. La producción de bienes de capital fue prácticamente nula. Además, la producción industrial dependía enormemente del mercado nacional. La capacidad para exportar fue muy limitada. En 1951 sólo se exportó el 16\% del output del sector manufacturero; excluyendo del mismo la producción de alimentos, bebidas y tabaco se exportó tan sólo un 6\% del output (O'Malley, 1992: 32, 33). Además, los problemas de ineficiencia del sector industrial eran cada vez más claros (Haughton, 1995: 31).

Los cincuenta fueron los últimos de la estrategia ISI. La producción industrial entre 1951y 1958 aumentó un $2,8 \%$ y el GDP algo menos del 1\%. Poniendo estos datos en el contexto europeo es fácil concluir que estos modestos aumentos de la producción industrial y del producto interior fueron posibles gracias a la recuperación económica de Europa, y no se debieron a la superación de los problemas económicos que comenzaron a ser visibles durante la Segunda Guerra Mundial. En efecto, el GDP p.c. de Irlanda comparado con la media europea era un 75\% en 1951, y en 1958 pasó a ser un 60\% (Haughton, 1995: 34). Los problemas de balanza de pagos no se superaron, y mostraron las enormes

\footnotetext{
${ }^{5} \mathrm{La}$ fuente de la que tomo los datos se refiere al GDP p.c. de Irlanda respecto a la media de la Unión Europea. En las fechas a que refieren estos datos, la Unión Europea no existía. Desconozco respecto a qué países se han calculado estas proporciones.
} 
RIS

REVISTA INTERNACIONAL DE SOCIOLOGIA

№ 31, Enero-Abril, 2002

JUAN RAFAEL MORILLAS MARTÍNEZ

dificultades y los problemas de modernización y competitividad del sector industrial. Su causa fue la dependencia del sector industrial de la importación de bienes de capital, debida a la incapacidad de la producción doméstica de alcanzar la capitalización y el nivel de desarrollo técnico necesarios para sustituir su importación por producción doméstica. A la vez, las dificultades para exportar fueron muy importantes. El $84 \%$ de la producción del sector manufacturero se destinó al mercado doméstico (O’Malley, 1992: 33).

Las crecientes dificultades experimentadas por la economía irlandesa y el reconocimiento de su relación con la estrategia ISI determinaron su abandono y la opción por una nueva estrategia de desarrollo industrial basada en la liberalización del comercio internacional y en la atracción de inversiones extranjeras directas. La escasez de capital, la debilidad tecnológica, las enormes dificultades para exportar la producción industrial, la dependencia del sector manufacturero de la importación de inputs, los problemas crónicos de balanza de pagos. Desde mediados de los años cincuenta, el Ministro de Hacienda, T.K. Whitaker, llevó a cabo una revisión de la política económica seguida hasta entonces. En 1958, el gobierno irlandés publicó el informe Economic Development, conocido como Informe Whitaker, en el que se recogían las conclusiones de dicha revisión. Sus propuestas eran la disminución del arancel y el establecimiento de incentivos para estimular la inversión industrial privada en sectores exportadores (Jacobsen, 1994: 66; O'Hearn, 1995: 92; Haughton, 1995: 35, 36; O'Sullivan, 1995: 364).

El esquema de política industrial en que se ha basado la nueva estrategia de desarrollo industrial ha sido básicamente el mismo desde finales de los años cincuenta, con leves diferencias debidas al énfasis en unos instrumentos o a la adición de algunas medidas concretas (O'Sullivan, 1995: 364, 365). Los principales instrumentos de este esquema son los siguientes:

1. Establecimiento de incentivos para conseguir que la industria doméstica orientara su producción a la exportación. Estos incentivos consistieron, y consisten, en el establecimiento de exenciones fiscales de los beneficios obtenidos por las exportaciones ${ }^{6}$. Se fortaleció el papel de la Industrial Development Authority (en adelante IDA), establecida en 1949, que fue la encargada de la concesión de las exenciones fiscales y de las subvenciones a las industrias orientadas a la exportación.

2. Atracción de FDIs. El mecanismo utilizado fue la extensión de las exenciones fiscales y de las subvenciones a las FDIs. Esta medida se adoptó

\footnotetext{
${ }^{6} \mathrm{La}$ exención fiscal de los beneficios de las exportaciones fue del $100 \%$ hasta 1980 . Desde 1981 el tipo impositivo aplicado a los beneficios de todas las industrias manufactureras es del 10\% (O'Malley, 1992: 39).
} 
esperando que las empresas creadas por las FDIs tendrían efectos beneficiosos para el sector industrial nacional gracias a las externalidades positivas que podrían ejercer sobre el mismo: creación de linkages entre ambos sectores, procesos de learning, etc.

3. Liberalización del comercio. Las cuotas fueron desapareciendo progresivamente durante los años cincuenta. Se redujo el arancel. En 1963 y 1964 Irlanda redujo unilateralmente los aranceles sobre los bienes producidos en el Reino Unido. En 1965 se creó la Anglo-Irish Free Trade Area. En 1967 suscribió el GATT. En 1973 se adhirió a la Comunidad Europea (CE); previamente, junto a la solicitud de adhesión a la CE en 1961, redujo el arancel con los países miembros.

\section{Consecuencias de la fase ELI}

Como se observa en la tabla 1 , la producción del sector manufacturero experimentó un importante crecimiento. En el período 1960-73 el crecimiento de la producción de manufacturas duplicó al de la década de los cincuenta (última fase de ISI). En el período siguiente la producción de manufacturas también creció, aunque a un ritmo menor, debido a factores de demanda (resultado de las consecuencias económicas de los años setenta y primera mitad de los ochenta). El importante crecimiento de la producción de manufacturas de la segunda mitad de los ochenta $(11,5 \%)$ fue consecuencia, además de la recuperación de la demanda, de factores de oferta asociados a la llegada de nuevas FDIs.

La evolución del crecimiento del empleo en el sector manufacturero experimentó un importante aumento respecto a la última fase de ISI, pero a partir de 1973 no conoció crecimientos significativos a pesar del espectacular

Tabla 1.

Crecimiento de la producción y del empleo en el sector manufacturero (\%)

\begin{tabular}{lccc}
\hline & Output & Empleo & Output/Empleo \\
\hline $1950-1959$ & 3,1 & 0,8 & 3,9 \\
$1960-1973$ & 6,5 & 2,3 & 2,8 \\
$1973-1979$ & 5,1 & 0,8 & 6,4 \\
$1980-1986$ & 4,1 & $-2,7$ & $-1,5$ \\
$1987-1989$ & 11,5 & 0,8 & 14,4 \\
$1990-1992$ & 6,0 & 1,0 & 6,0 \\
\hline
\end{tabular}

Fuente: O`Sullivan (1995: 366). 
RIS

REVISTA INTERNACIONAL DE SOCIOLOGÍA

№ 31, Enero-Abril, 2002

JUAN RAFAEL MORILLAS MARTÍNEZ

crecimiento de la producción del sector manufacturero ${ }^{7}$. Entre 1973 y 1979, el empleo en este sector creció a un modesto ritmo del $0,8 \%$, mientras el output lo hizo a un 5,1\%. En la primera mitad de los ochenta, aunque la producción siguió aumentando el empleo decreció un 2,7\%. El espectacular crecimiento de la producción a finales de los ochenta estuvo acompañado de un muy modesto crecimiento del empleo $(0,8 \%)$, relación que coincide con la del último período incluido en la tabla.

El motor del crecimiento de la producción de manufacturas y del empleo en el mismo fue el sector de empresas de propiedad extranjera. En efecto, la mayoría de las ayudas concedidas por la IDA fue tomada por empresas de propiedad no-irlandesa (O'Sullivan, 1995: 366; Jacobsen, 1994: 70 y ss.). El sector doméstico, dada su incapacidad de competir, tanto en los mercados internacionales como en el mercado nacional con los bienes importados, tuvo una participación escasa e incluso negativa en el crecimiento del empleo. $\mathrm{La}$ evolución del empleo en este sector aumentó en los años cincuenta y hasta la mitad de los sesenta. Desde 1966 no aumentó y disminuyó en los ochenta. Esta evolución demuestra la incapacidad de competir del sector doméstico. El empleo aumentó mientras que la industria doméstica disfrutó de protección, hecho que ocurrió hasta mediados de los sesenta. Desde entonces se mantuvo constante gracias a la demanda doméstica. Cuando ésta se debilitó en los años ochenta, la competencia internacional no encontró ninguna fuerza que contrarrestara su influencia y, de este modo, el empleo disminuyó. Los cambios estructurales experimentados por la industria doméstica confirman esta interpretación. Los únicos subsectores en que ha aumentado el empleo han sido aquellos en que existe un alto grado de protección natural: productos primarios, bienes no susceptibles de comercialización en los mercados internacionales y productos que requieren altos costes de transporte. Los sectores donde tienen mayor importancia las economías de escala y las tecnologías disponibles han experimentado una importante disminución de empleo y del número de empresas, consecuencia de sus problemas de competitividad (O'Malley, 1992: 34-38).

Los datos de la tabla 2 muestran que la disminución de empleos en el sector manufacturero durante los años ochenta, además de los periodos de segunda mitad de los setenta y primera de los noventa, se concentró en el sector doméstico. La estructura y composición del empleo en el sector manufacturero, dada la diferente especialización de los sectores doméstico y extranjero -en

\footnotetext{
${ }^{7}$ Un análisis completo de la evolución del empleo en Irlanda debería contemplar no sólo factores de demanda, sino también los determinantes de la oferta de trabajo, dada la importancia de las altas tasas natalidad y de emigración de la población de Irlanda en el funcionamiento del mercado de trabajo.
} 
Tabla 2.

Cambios en el empleo industrial en los sectores doméstico y extranjero

\begin{tabular}{lcc}
\hline & Sector doméstico & Sector extranjero \\
\hline Empleo total 1973 & $145.815(67,8 \%)$ & $69.388(32,2 \%)$ \\
Empleo total 1994 & $114.613(56,0 \%)$ & $90.099(44,0 \%)$ \\
Diferencia & $-31.202(-21,4 \%)$ & $20.711(+29,9 \%)$ \\
\hline
\end{tabular}

Fuente: O'Sullivan (1995: 369).

actividades tradicionales, el primero, y en actividades intensivas en nuevas tecnologías el segundo-, abunda en esta conclusión. Los datos presentados por O'Sullivan (1995: 370) muestran reducciones del empleo en los sectores tradicionales y aumentos en los modernos, controlados éstos por FDIs.

Así pues, el sector de empresas de propiedad extranjera es el principal responsable de la evolución del crecimiento de la producción de manufacturas y de la evolución del empleo. El examen de sus efectos requiere distinguir dos fases diferentes en la estrategia ELI. Durante los años sesenta, las FDIs se dirigieron a sectores con un nivel medio de desarrollo tecnológico e intensivos en trabajo (textil, plásticos e ingeniería eléctrica). En los años setenta la estrategia de la IDA consistió en la atracción de FDIs en sectores de alta tecnología, y las subvenciones que concedía favorecieron a las inversiones intensivas en capital frente a las inversiones intensivas en trabajo (Jacobsen, 1994: 112; O'Malley, 1992: 39). Así, desde la primera mitad de los setenta el desarrollo tecnológico del sector moderno ha aumentado. Su composición está dominada por cinco sectores principales: electricidad y electrónica, maquinaria, productos farmacéuticos, productos médicos y bienes de equipo. Estos sectores han sido los principales responsables del crecimiento de la producción industrial, del aumento de las exportaciones y de la evolución del empleo. El contenido de factores de la producción de estos sectores es intensivo en capital y en trabajo cualificado. Los linkages establecidos por estos sectores con los domésticos han sido escasos. La mayoría de los inputs que utilizan en sus procesos de producción son importados ${ }^{8}$ (O'Sullivan, 1995: 388; Jacobsen, 1994:110; O'Malley, 1992: 39, 40).

\footnotetext{
${ }^{8}$ La principal causa de esta característica es la influencia de los procesos de integración vertical de la producción de las empresas multinacionales. Feenstra y Hanson (1996) llaman outsourcing al proceso de traslado de algunas fases del proceso de producción a países en desarrollo. Estos autores destacan las consecuencias distributivas de los procesos de outsourcing.
} 
RIS

REVISTA INTERNACIONAL DE SOCIOLOGIA

№ 31, Enero-Abril, 2002

Los datos de la ratio crecimiento del ouput-crecimiento del empleo presentados en la tercera columna de la tabla 1 revelan la enorme productividad del sector moderno, consecuencia de su intensidad en capital. En el período 1960-73 el crecimiento del empleo en un 1\% requería un crecimiento del output de un $2,8 \%$. Entre 1974 y 1979 requería un crecimiento del output del $6,4 \%$. En el período 1980-86, aunque el output creció, el empleo disminuyó. Entre 1987 y 1989 fue necesario un aumento de 14,4 puntos del output para que el empleo creciera un punto. En 1990-92, fue necesario un 6\%.

La diferente orientación de las FDIs en los años sesenta y setenta es fundamental para comprender la evolución de la relación output-empleo y de las consecuencias distributivas de la estrategia ELI. En ambos períodos, el sector extranjero fue el responsable del crecimiento del output del sector manufacturero y de la evolución del empleo. Desde mediados de los sesenta, el empleo en el sector doméstico no aumentó, y en los ochenta disminuyó. La menor relación output/empleo del período 1960-1973 respecto a los períodos posteriores se debe al diferente carácter de las FDIs. En el primero, las FDIs demandaron trabajo de un modo considerable. Desde los años setenta, las FDIs se dirigieron a actividades mucho más intensivas en capital, y sólo ha demandado trabajo cualificado. Esta es la razón de los altos valores de la ratio output/empleo y de que sea posible que, como ocurrió en la primera mitad de los ochenta, un importante crecimiento de la producción del sector manufacturero esté acompañada con una disminución del empleo'.

Los datos recientes de crecimiento económico y de evolución del empleo confirman las relaciones expuestas. El crecimiento del PIB real en Irlanda en el periodo 1990-93 fue del 4,4\%, mientras en los países de la OCDE creció un $1,7 \%$. En el período $1994-97$ creció un $7,7 \%$ frente a un 2,8\% (media de los países de la $\mathrm{OCDE}^{10}$ ). Aunque la recuperación de la demanda ha influido en

\footnotetext{
${ }^{9} \mathrm{La}$ productividad media del sector manufacturero irlandés en 1987 fue del $137 \%$ (un 16\% mayor que en el Reino Unido y un $20 \%$ mayor que en Alemania) (cálculos propios a partir de O'Sullivan, 1995: 371). A.E. Murphy (1998: 6), basándose en datos del Censo de Producción Industrial de Irlanda de 1995, calcula el output y el empleo de los sectores moderno y doméstico de Irlanda. A partir sus resultados, he realizado unos cálculos que han revelado que la producción por trabajador empleado es 8,7 veces mayor en los cinco sectores high-tech, que en el sector manufacturero doméstico. Estos valores tan elevados de la productividad de las empresas multinacionales en Irlanda son puestos en duda por muchos economistas, que señalan que son consecuencia de las prácticas contables de Transfer-Pricing de las multinacionales, consistentes en atribuir a su producción en Irlanda la producción obtenida en otros países, debido al bajo tipo impositivo irlandés sobre los beneficios de industrias orientadas a la exportación.

${ }^{10}$ Cálculos de A. Coughlan (1998: 1) a partir de datos de OECD, Economic Outlook, junio 1997. Los datos de 1997 son estimados.
} 
Tabla 3.

Tasa de desempleo y desempleo de larga duración (\%)

\begin{tabular}{lcccccc}
\hline & 1991 & 1992 & 1993 & 1994 & 1995 & 1996 \\
\hline Tasa de desempleo & 14,7 & 15,1 & 15,7 & 14,8 & 12,2 & 11,9 \\
Desempleo larga duración & 59,4 & 56,3 & 56,9 & 60,7 & 58,2 & 57,6 \\
\hline
\end{tabular}

Fuente: OECD (1997: 117).

este importante crecimiento económico (OECD, 1997; O'Leary, 1998), la influencia de los factores de oferta ha sido determinante para que los niveles de crecimiento sean tan espectaculares (The Economist,1997; IMF, 1997; OECD, 1997; Coughlan, 1998). La principal causa ha sido el aumento de las FDIs y el incremento de las exportaciones de los cinco sectores high-tech. A.E. Murphy ha calculado que las exportaciones de estos cinco sectores han sido las responsables del $60 \%$ del crecimiento del PIB nominal. Entre 1990 y 1996, el PIB nominal creció un 55\%; si se excluye las exportaciones de los cinco sectores, el crecimiento hubiera sido del 23\% (Murphy, 1998: 6).

Los datos de la tabla 3 muestran que la tasa de desempleo experimentó una reducción significativa, aunque leve, en 1995. Los niveles de desempleo de larga duración son especialmente altos. El crecimiento económico, aunque ha reducido de un modo modesto la tasa de desempleo, casi no ha afectado a la tasa de desempleo de larga duración. Disminuyó en 1992, pero aumentó significativamente en 1994, y aunque se redujo en 1995, sólo lo hizo en dos puntos. En el período 1980-1987, que fue un período en que aumentó el desempleo, además de la emigración, el desempleo de larga duración aumentó un $9 \%$. Según datos de estadísticas de desempleo registrado, la proporción de desempleados de larga duración respecto al total de desempleados fue del $35 \%$ en abril del 1980. En abril de 1987 fue del 44\%. En 1991, según los datos de la tabla anterior, fue del 59,4\%. En 1992, el 44\% de los desempleados lo estuvieron durante más de tres años (Whelan, 1996: 33).

Este gap entre crecimiento económico y evolución de la tasa de desempleo y de la tasa de desempleo de larga duración se debe a que la causa del crecimiento no tiene efectos importantes sobre la evolución del empleo, y su influencia sobre la evolución del empleo no cualificado es prácticamente nula. La principal causa de este crecimiento económico ha sido el aumento de las exportaciones de los cinco sectores high-tech. El contenido de factores de estas exportaciones es capital (procedente de FDIs) y trabajo cualificado. La intensidad de capital de estos sectores permite importantes aumentos de la producción, acompañados de aumentos 
RIS

REVISTA INTERNACIONAL DE SOCIOLOGIÁ

№ 31, Enero-Abril, 2002

JUAN RAFAEL MORILLAS MARTÍNEL

Tabla 4.

Tasa de desempleo y desempleo de larga duración (\%)

\begin{tabular}{lcrr}
\hline & 1997 & 1998 & 1999 \\
\hline Tasa de desempleo & 10,3 & 7,8 & 5,7 \\
Desempleo larga duración & 54,3 & 50,2 & 42,9
\end{tabular}

Fuente: O'Connell (2000: 70).

de la demanda de trabajo cualificado, sin afectar a la demanda de trabajo no cualificado ${ }^{11}$.

En la tabla 4 se muestra datos más recientes de la evolución de la tasa de desempleo y de la proporción de desempleo de larga duración. Estos datos muestran una reducción de ambas en el período 1997-99.

La evolución de la tasa de desempleo y del desempleo de larga duración parecen contradecir la conclusión anterior. En concreto, la reducción del desempleo de larga duración parece ser consecuencia de la extensión de las consecuencias del crecimiento económico a los trabajadores no cualificados. Ciertamente, existe una asociación entre el crecimiento económico de los últimos años (que ha alcanzado el $8 \%$ anual en la segunda mitad de los años noventa) y la reducción del desempleo de larga duración. Ahora bien, ¿existe una relación de causalidad entre ambas variables? ¿Se ha traducido el crecimiento económico en un aumento de la demanda de trabajadores no-cualificados?.

La respuesta a estas preguntas es no. La disminución del desempleo en la segunda mitad de los años noventa ha sido consecuencia principalmente de la reducción del desempleo de larga duración (O'Connell, 1998). Y la reducción de éste ha tenido escasa relación con el crecimiento económico. La evolución del desempleo de larga duración ha sido una consecuencia de los programas públicos de contratos de formación y de contratos temporales orientados a los trabajadores no cualificados (O’Connell, 1998, 2000).

Además, esta reducción de la proporción de desempleados de larga duración se debe en gran parte a que los trabajadores no cualificados han dejado de estar

\footnotetext{
${ }^{11}$ La excesiva dependencia de las FDIs, los escasos linkages establecidos entre ellas y el sector doméstico y la necesidad de fomentar el sector industrial doméstico fueron reconocidos por el National Economic and Social Council en el informe Industrial Policy. Desde mediados de los ochenta se han adoptado ciertas medidas para fomentar el desarrollo tecnológico del sector industrial doméstico (O'Malley, 1992: 43, 44). No dispongo de datos para evaluar sus resultados sobre el sector doméstico. En cualquier caso, la dependencia del crecimiento económico de las FDIs continúa siendo un factor característico de la economía de Irlanda.
} 
desempleados temporalmente gracias a los programas públicos de contratos de formación. Una perspectiva estática muestra que el desempleo de larga duración ha disminuido. Pero una perspectiva dinámica muestra que tal desempleo ha sido sustituido por una rotación del desempleo de larga duración a contratos de formación y contratos temporales, y de ahí vuelta al desempleo (O'Connell, 1998). La mayor parte de los trabajadores que inicialmente estaban desempleados durante más de un año y han participado en un programa público de empleo, han vuelto a estar desempleados una vez concluido su contrato. Por tanto, una considerable proporción de trabajadores no-cualificados no ha estado empleada continuamente, sino que ha experimentado un proceso circular. Ha transitado del desempleo de larga duración al empleo en los citados programas públicos de empleo, y una vez finalizados sus contratos han vuelto al desempleo. Pero, al haber estado ocupados previamente, son desempleados de corta duración, por lo cual, aunque vuelven a estar desempleados, ya no son contabilizados como parados de larga duración.

En conclusión, debido a que el crecimiento económico de la estrategia ELI de Irlanda se ha basado en la exportación de bienes cuyo contenido de factores ha sido esencialmente capital y trabajo cualificado, los beneficios del crecimiento económico no se han extendido por igual a toda la población. En la siguiente sección analizo las consecuencias distributivas de tal estrategia de desarrollo a partir del modelo analítico de la sección anterior y de un análisis empírico de la distribución de los salarios.

\section{Las consecuencias distributivas de la fase ELI}

\section{Análisis a la luz del modelo analítico}

A continuación paso a analizar las consecuencias distributivas de la estrategia ELI a partir del modelo analítico que presenté en el segundo epígrafe. Me centro en la fase posterior a 1973. Como se concluye de la descripción anterior, esta fase se puede simplificar para ser estudiada del modo presentado en la sección anterior. Primero, estudio las influencias sobre la distribución funcional de la renta y, estimando la propiedad de los factores, paso a analizar la influencia sobre la tendencia que sigue la distribución personal de la renta de los propietarios de cada factor.

\section{Efectos sobre el capital ( $\mathrm{j}=1)$}

La renta del capital está determinada por la siguiente expresión:

$$
w_{1}=w_{1}\left(F_{11}, F_{2 l} ; L_{11}, L_{2 l}\right)
$$

Donde $F_{11}<F_{2 l}$, es decir, que la productividad marginal del capital es menor en el sector tradicional que en el sector moderno de la economía. 
RIS

REVISTA INTERNACIONAL DE SOCIOLOGIA

No 31, Enero-Abril, 2002

JUAN RAFAEL MORILLAS MARTÍNEL

Aunque la productividad marginal del capital es menor en el sector tradicional, lo cual podría hacer disminuir la renta media de este factor, como $L_{11}<<L_{21}$ (es decir, que el capital empleado en el sector tradicional es mucho menor que el empleado en el sector moderno, puesto que las FDIs han supuesto la mayor parte del crecimiento del stock de capital) la diferencia de productividad marginal intersectorial no determina una disminución significativa de la renta media del capital.

Supongo que la economía emplea toda la dotación de capital existente ${ }^{12}$; o sea que $L_{11}+L_{12}=L_{1}=D_{1}=\sum a_{i 1}$.

Respecto a la propiedad $a_{i l}$ se da una concentración muy alta en el sector moderno y menor concentración en el sector tradicional.

Respecto a la distribución personal de la renta, y según la expresión (1) o (5) presentada en el anterior epígrafe, un grupo reducido de personas recibirá una renta elevada, siendo mucho mayor la concentración y el volumen de renta recibida en el caso del capital empleado en el sector moderno.

\section{Efectos sobre el trabajo cualificado $(j=2)$}

La demanda y la oferta de trabajo cualificado han aumentado desde los años setenta $^{13}$. Las exportaciones del sector moderno, consistentes en productos tecnológicamente avanzados, han sido las principales responsables del crecimiento económico. El contenido de factores de estas exportaciones es, además de capital, trabajo cualificado.

Supongo que sólo el sector moderno emplea trabajo cualificado, y que la demanda de éste coincide con la dotación total del mismo. Así, tenemos que:

$$
\begin{gathered}
w_{2}=w_{2}\left(F_{12}, F_{22} ; L_{12}, L_{22}\right) \text { donde } F_{12}=0 \text { y } L_{12}=0 \\
L_{22}=L_{2}=\sum a_{i 2}=D_{2}
\end{gathered}
$$

La renta recibida por este factor es media-alta debido a que su productividad marginal es alta en el sector moderno, a que todo el empleo efectivo de este

\footnotetext{
${ }^{12}$ Para que esta formalización fuera más acorde a la realidad sería necesario comprobar si se utiliza todo el capital disponible en el sector doméstico. En cualquier caso, aunque exista sub-empleo en este sector, la renta media del capital será muy alta debido a que la cantidad de capital empleada en el sector doméstico es mucho menor que la que emplea el sector moderno.

${ }^{13}$ Según datos de Whelan, Breen y Whelan (1992: 108 y 109) el crecimiento de las ocupaciones no manuales en la estructura ocupacional de Irlanda entre 1971 y 1985 fue superior al $40 \%$. Antes de 1961 era un grupo prácticamente inexistente; comenzó a crecer en los años sesenta y experimentó su mayor expansión en los años setenta.
} 
factor tiene lugar en el sector moderno de la economía, y a que no sufre subempleo.

\section{Estructura de la propiedad del factor $j=2$}

Una importante proporción de la población activa tiene altos niveles de cualificación, como consecuencia de las altas tasas de natalidad en periodos anteriores - lo que implica la disponibilidad de una alta proporción de población joven-y de los altos niveles educativos, especialmente de la población más joven ${ }^{14}$. Por tanto, es un grupo numeroso cuya renta, que es media-alta, está distribuida de un modo homogéneo. Por ello, contribuye a aumentar la igualdad en la distribución de la renta.

\section{Efecto sobre el trabajo no cualificado $(\mathrm{j}=3$ )}

La demanda de trabajo no cualificado ha disminuido - consecuencia de los problemas del sector tradicional de la economía, expuestos en las páginas anteriores-, y su oferta, aunque también ha disminuido, lo ha hecho en menor medida $^{15}$. El resultado ha sido el subempleo de este factor: $L_{23}=\sum a_{i 3}<D_{3}$. Supongo que sólo el sector tradicional emplea trabajo no cualificado. La renta de este factor está determinada por $w_{3}=w_{3}\left(F_{13}, F_{23} ; L_{13}, L_{23}\right)$, siendo $F_{23}=0$ y $L_{23}=0$. La baja productividad marginal del trabajo no cualificado en el sector tradicional y el subempleo del mismo determinan que la renta de este factor sea baja.

\section{Estructura de la propiedad del factor "trabajo no cualificado" $\left(\mathrm{a}_{\mathrm{i} 3}\right)$}

Aunque la proporción de la población con bajo nivel de cualificación ha disminuido, continúa siendo un grupo numéricamente importante. Las características de la renta de este factor y la estructura de la propiedad del mismo imprimen una tendencia de aumento de la desigualdad en la distribución personal de la renta, pues constituye un grupo numéricamente importante que recibe una porción baja de la renta.

\footnotetext{
${ }^{14}$ Datos de Whelan et al. (1992: 108 y 109) muestran que en 1970 el $70 \%$ de la población mayor de quince años permanecía en el sistema educativo; en 1985 aumentó al $94 \%$.

${ }^{15}$ Desde 1961 el desempleo de los trabajadores no cualificados ha sido mayor del $30 \%$ (Whelan et al., 1992: 118).
} 
RIS

REVISTA INTERNACIONAL DE SOCIOLOGíA

№ 31, Enero-Abril, 2002

Tendencias de la distribución de la renta y de la desigualdad

Es imposible con la información que he presentado ofrecer un valor de la desigualdad de la renta debida a la apertura comercial internacional y a la inversión extranjera directa. El modelo analítico desarrollado no ofrece explicación de las relaciones entre todas las variables que intervienen en esta relación. Además, no he presentado datos de la estructura de la propiedad de los factores; sólo he realizado unas estimaciones apresuradas. Ahora bien, es posible discutir a partir del modelo presentado y de su aplicación en las líneas anteriores las posibles tendencias experimentadas por la distribución de la renta como consecuencia de la estrategia ELI seguida por Irlanda desde 1973.

Los efectos de esta estrategia sobre el trabajo cualificado han determinado una mejora de la distribución de la renta. La oferta de este factor han aumentado, como consecuencia de la mejora de los niveles de formación de la población. La demanda también ha aumentado. El motor del crecimiento económico han sido las exportaciones del sector moderno de la economía, y éstas tienen un alto contenido de trabajo cualificado.

Los efectos sobre el capital introducen una tendencia al aumento de la desigualdad. La alta renta que recibe este factor, y la alta concentración de su propiedad, determinan que un grupo muy reducido de personas concentre una porción extremadamente alta de renta. Las diferencias de renta del capital según el sector en que está empleado determinan un alto grado de aumento de la desigualdad entre ambos segmentos del capital. El capital en el sector tradicional ha empeorado su situación con la estrategia ELI. La protección de que disponía en la fase ISI le permitía controlar el mercado doméstico. La inexistencia de linkages entre los sectores tradicional y moderno, la incapacidad de competir exportando los bienes que produce al mercado internacional y la incapacidad de competir con la importación de bienes determinan un empeoramiento de su situación en esta fase de apertura comercial y recepción de FDIs.

El proceso secular de transformación económica de Irlanda, consecuencia de su rápida y tardía industrialización, ha provocado una transformación de la estructura ocupacional, consistente en una disminución de las antiguas oportunidades de empleo para los trabajadores no cualificados, que no han sido sustituidas por otras de nueva creación. Estudios de movilidad social han revelado que, aunque la experiencia de Irlanda ha sido similar a la de los países occidentales, ha experimentado una tendencia particular: la existencia de barreras a la movilidad social de la clase trabajadora industrial. La homogeneidad social de este grupo (el origen social del $90 \%$ de sus componentes es el de trabajadores industriales no cualificado y trabajadores agrícolas) revela las enormes dificultades de movilidad social ascendente de este grupo. Su situación socioeconómica es muy desfavorable. El desempleo de larga duración está compuesto casi en su totalidad por trabajadores no cualificados. El $40 \%$ de los 
trabajadores no cualificados estaba en situación de pobreza en $1987^{16}$ (Whelan, et al., 1992: 125-127). Las actividades fomentadas por las FDIs no han generado una demanda significativa de trabajo no cualificado, tanto de un modo directo como de un modo indirecto - a través del establecimiento de linkages con el sector tradicional.

Resumiendo, las principales tendencias distributivas de la estrategia ELI han sido las siguientes. 1) Ha mejorado la renta del trabajo cualificado. La importancia numérica de este grupo determina que el incremento de renta de este factor no esté concentrado y, por tanto, imprime una tendencia igualitaria a la distribución personal de la renta. 2) Los efectos sobre el trabajo no cualificado han sido muy diferentes. El subempleo de este factor, la limitación del empleo del mismo al sector tradicional y su baja productividad marginal han determinado que la renta apropiada por este factor sea muy reducida. La estrategia ELI no sólo no ha mejorado la renta de este factor, sino que el proceso de cambio estructural de la economía asociado a la misma ha tenido como consecuencia una disminución de oportunidades de empleo de este factor. 3) Esta estrategia de industrialización ha producido una alta concentración de la renta personal de los propietarios del capital empleado en el sector moderno de la economía. La alta parte de renta apropiada por este factor y la alta concentración de la propiedad del mismo son los causantes. Ahora bien, como la mayoría de este capital es de propiedad extranjera, su efecto sobre la distribución personal de la renta, calculada para los nacionales irlandeses, es muy limitado ${ }^{17}$. La influencia sobre el capital empleado en el sector doméstico ha sido diferente. La eliminación de la protección de la industria doméstica determinó el estancamiento de la misma.

\section{Evidencia empirica de las consecuencias distributivas de la estrategia ELI}

En la tabla 5 se presentan datos de la distribución salarial de la población activa en el período 1987-97. Estos datos muestran lo siguiente: 1) la proporción respecto al salario mediano que han representado los deciles y quartiles inferiores

$$
\text { de vida. }
$$

${ }^{16} \mathrm{El}$ indicador de pobreza que utilizan estos autores combina medidas de renta y de estilo

${ }^{17} \mathrm{La}$ renta de los factores de propiedad extranjera ha aumentado mucho. Los datos de Contabilidad Nacional disponibles reflejan el siguiente crecimiento. Tomando como base el año 1986 (1986=100), la renta de los factores extranjeros ha aumentado desde entonces, experimentando los máximos crecimientos a partir de 1992. Era 172 en 1990; 167 en 1991; 179 en 1992; 204 en 1993 y 220 en 1994. Los mayores aumentos de este índice coinciden con el espectacular crecimiento económico de Irlanda desde 1992, basado en el aumento de las FDIs y de las exportaciones (Cálculo propio a partir de OECD: 1996). 
Tabla 5 .

Distribución de los salarios/hora en 1987, 1994 y 1997

(Proporción respecto al salario mediano)

\begin{tabular}{lrrr}
\hline & 1987 & 1994 & 1997 \\
\hline Percentil 99 & 3,6 & 3,8 & 4,3 \\
Percentil 95 & 2,4 & 2,8 & 3,0 \\
Decila superior & 1,96 & 2,24 & 2,33 \\
Quartil superior & 1,37 & 1,50 & 1,53 \\
Decila inferior & 0,47 & 0,47 & 0,48 \\
Quartil inferior & 0,73 & 0,68 & 0,69 \\
Ratio Perc 90/Perc 10 & 4,16 & 4,77 & 4,81 \\
\hline
\end{tabular}

Fuentes: Para los datos de 1987: Economic and Social Research Institute Household Surveys; para los datos de 1994 y 1997: Living in Ireland Survey y Barret, Fitz Gerald y Nolan (2000: 130).

y superiores de la distribución, así como la proporción respecto al salario mediano de los percentiles 95 y 99 de la distribución, y 2) el ratio entre el salario recibido por la decila superior y la decila inferior de la distribución.

Los datos de la tabla anterior muestran que la distribución salarial ha sido más dispersa en 1994, que en 1987, y en 1997 más que en 1984. La dispersión salarial ha aumentado durante este período. Pero lo ha hecho más en el subperíodo que va de 1987 a 1994, que en el que transcurre entre 1994 y 1997. El ratio del salario de la decila superior al salario de la decila inferior aumentó un $15 \%$ en el período 1987-94, y un 16\% en el período 1987-97.

Los datos de la tabla anterior muestran también que los salarios en la parte inferior de la distribución no han crecido en la década 1987-97, mientras que sí lo han hecho los salarios en la parte superior de la distribución. Los salarios en la decila inferior se han mantenido constantes durante todo el período, mientras que los de la decila superior experimentaron un incremento del $14 \%$ en el período 1987-94, y del 19\% en el período 1987-97. Si analizamos la parte más superior de la distribución salarial, observamos igualmente un crecimiento del salarial respecto al salario medio. Así, la proporción del valor de los percentiles 95 y 99 de la distribución de los salarios respecto al salario mediano ha aumentado durante todo el período.

Estos datos muestran, en conclusión, que las consecuencias distributivas de la estrategia ELI en Irlanda no han tenido el mismo signo para todos los grupos sociales. Hay grupos que han mejorado su situación, mientras otros se han mantenido igual. Los trabajadores cualificados han aumentado su salario, mientras que los de la parte inferior de la distribución no han experimentado ningún progreso. 


\section{CONCLUSIONES}

Si se puede obtener alguna conclusión del estudio de caso realizado, ésta es la complejidad de las consecuencias distributivas de las estrategias ELI. El simple conocimiento de la dotación de factores de la economía y la consiguiente orientación de las exportaciones, conforme a una interpretación basada en la teoría Heckscher-Ohlin, no permiten conocer el signo de los efectos distributivos. Es necesario examinar la influencia de más variables. Hay que considerar aspectos de organización industrial, la orientación de las FDIs, la intensidad de capital de las exportaciones, la composición de la estructura de la demanda de trabajo, los cambios experimentados por las estructuras socioeconómicas y ocupacional o el carácter de las exportaciones e importaciones. Una larga letanía que no se reduce a la dotación de factores y a la especialización de las exportaciones.

Obviamente, este hecho no se debe a que la teoría Hecksher-Ohlin no sea válida ${ }^{18}$. Como toda teoría, parte de unos supuestos y establece una relación entre dos grupos de variables: causas y efectos. La utilización de la teoría es válida cuando la situación a que se aplica se acomoda a las condiciones establecidas en los supuestos. Como la realidad es más compleja que los supuestos, se realizan simplificaciones. La aplicación de la teoría será válida si se puede asumir las simplificaciones realizadas. En el caso de la teoría Heckscher-Ohlin, la complejidad de las experiencias ELI no permite su aplicación de forma indiscriminada.

\footnotetext{
${ }^{18} \mathrm{La}$ evolución de ciertas variables parece seguir la línea predicha por esta teoría. El proceso de integración internacional de la economía irlandesa ha influido en la convergencia de los costes laborales entre Irlanda y el Reino Unido (Curtis y Fitz Gerald, 1996). Este hecho es coherente con el aumento del precio de los factores implicados en el comercio internacional que predice la teoría Heckscher-Ohlin.
} 


\section{REFERENCIAS BIBLIOGRÁFICAS}

BARRET, A., J. FITZ GERALD y B. NOLAN (2000), "Earnings inequality, returns to education and low pay", en B. Nolan, P. O'Connell y C. Whelan (coords.), Bost to Boom? The Irish Experience of Growth and Inequality, Dublin, Institute of Public Administration.

BERRY, A. (1992), "Firm (or Plant) Size in the Analysis of Trade and Development", en G. Helleiner (coord.), Trade Policy, Industrialization, and Development, Oxford, Clarendon Press.

BOURGIOUN, F. y C. MORRISSON (1989), External Trade and Income Distribution, París, OECD.

CALLAN, T. y B. NOLAN (1992), "Income Distribution and Redistribution: Ireland in Comparative Perspective", en J. Goldthorpe y C. Whelan (coords.), The Development of Industrial Society in Ireland, Oxford, Oxford University Press.

COUGHLAN, A. (1998), "Some Notes on the Recent Economic Performance of the Irish Economy", Dept. of Social Studies (TCD), Dublin (manuscrito).

CURTIS J. y J. FITZ GERALD (1996), "Real Wage Convergence in an Open Labour Market", The Economic and Social Review, $\mathrm{n}^{\circ}$ 27, pp. 321-40.

ECONOMIC AND SOCIAL RESEARCH INSTITUTE (1987), Household Surveys, Dublín, ESRI.

FEENSTRA, R. y G. HANSON (1996), "Globalization, Outsourcing, and Wage Inequality", American Economic Review, $\mathrm{n}^{\circ}$ 86, pp. 240-245.

FIELDS, G. (1988), "Income Distribution and Economic Growth", en G. Ranis y T. Schultz (coords.), The State of Development Economics, Oxford, Basil Blackwell.

FINDLAY, R. (1988), "Trade, Development, and the State", en Ranis y Schultz (coords.), The State of Development Economics, Oxford, Basil Blackwell.

FREEMAN, R. (1995), “Are your wages set in Beijing?”, Journal of Economic Perspectives, $\mathrm{n}^{\circ}$ 9, pp. 15-32.

HAGGARD, S. (1990), Pathways from the Periphery, Ithaca, Cornell University Press.

HAUGHTON, J. (1995), “The Historical Background”, en J. O'Hagan (coord.), The Economy of Ireland, London, MacMillan.

HELLEINER, G. (1992), "Introduction", en G. Helleiner (coord.), Trade Policy, Industrialization, and Development, Oxford, Clarendon Press.

IMF (1997), World Outlook, Washington, DC, IMF.

JACOBSEN, J. (1994), Chasing Power in the Irish Republic, Cambridge, Cambridge University Press. 
KENNEDY, K. (1992), "The Context of Economic Development", en J. Goldthorpe y C. Whelan (coords.), The Development of Industrial Society in Ireland, Oxford, Oxford University Press.

KRUEGER, A. (1988), “The Relationships Between Trade, Employment, and Development”, en G. Ranis y T. Schultz (coords.), The State of Development Economics, Oxford, Basil Blackwell.

KRUGMAN, P. y M. OBSTFELD (1995), Economia Internacional, Madrid, McGraw-Hill.

KUZNETS, S. (1955), "Economic Growth and Income Inequality", American Economic Review, $\mathrm{n}^{\circ} 45$, pp. 1-28.

LAWRENCE, R. (1996), Single World, Divided Nations?, París, OECD.

LEE, N. (1992), "Market Structure and Trade in Developing Countries", en G. Helleiner (coord.), Trade Policy, Industrialization, and Development, Oxford, Clarendon Press.

MURPHY, A. (1998), "Beneath Celtic Tiger mask is US high-tech face", The Irish Times, 30 de enero.

O'CONNELL, P. (1998), "Spending Priorities in Labour Market Policies for the Unemployed", en ESRI, Budget Perspectives, Dublín, ESRI.

(2000), "The Dynamics of the Irish Labour Market in Comparative Perspective", en B. Nolan, P. O'Connell y C. Whelan (coords.), Bost to Boom? The Irish Experience of Growth and Inequality, Dublín, Institute of Public Administration.

O'HEARN, D. (1995), "Global Restructuring and the Irish Political Economy", en P. Clancy, S. Drudy, K. Lynch y O'Dowd, Irish Society. Sociological Perspectives, Dublín, Institute of Public Administration.

O'LEARY, J. (1998), “Flourishing like never before”, Sunday Independent, 17 de enero.

O'MALLEY, E. (1992), "Problems of Industrialization in Ireland", en J. Goldthorpe y C. Whelan (coords.), The Development of Industrial Society in Ireland, Oxford, Oxford University Press.

O'SULLIVAN, M. (1995), "Manufacturing and Global Competition”, en J. O'Hagan (coord.), The Economy of Ireland, London, MacMillan.

OECD (1996), National Accounts. Vol. III., París, OECD.

(1997a), OECD Economic Surveys. Ireland, París, OECD.

(1997b), Employment Outlook, París, OECD.

PACK, H. (1992), "Learning and Productivity Change in Developing Countries", en G. Helleiner (coord.), Trade Policy, Industrialization, and Development, Oxford: Clarendon Press. 
RIS

REVISTA INTERNACIONAL DE SOCIOLOGIA

№ 31, Enero-Abril, 2002

JUAN RAFAEL MORILLAS MARTÍNEZ

STEWART, F. y E. GHANI (1992), "Externalities, Development, and Trade", en G. Helleiner (coord.), Trade Policy, Industrialization, and Development, Oxford, Clarendon Press.

THE ECONOMIST (1997), “Europe's Tiger Economy”, 17 de mayo.

WHELAN, C. (1996), "Marginalization, Deprivation, and Fatalism in the Republic of Ireland: Class and Underclass Prespectives", European Sociological Review, n 12, pp. 33-51.

WHELAN, C., R. BREEN y B. WHELAN (1992), "Industrialization, Class Formation and Social Mobility in Ireland", en J. Goldthorpe y C. Whelan (coords.), The Development of Industrial Society in Ireland, Oxford, Oxford University Press. 